\title{
The Australasian Virtual Herbarium (AVH) and the Changing Role of Herbaria
}

\author{
Niels Klazenga $\ddagger$ \\ ‡ Royal Botanic Gardens Victoria, Melbourne, Australia
}

Corresponding author: Niels Klazenga (niels.klazenga@rbg.vic.gov.au)

Received: 16 Apr 2018 | Published: 15 Jun 2018

Citation: Klazenga N (2018) The Australasian Virtual Herbarium (AVH) and the Changing Role of Herbaria.

Biodiversity Information Science and Standards 2: e25866. https://doi.org/10.3897/biss.2.25866

\begin{abstract}
Australia's Virtual Herbarium (AVH) was created in 2001 and developed between 2001 and 2006 with the databasing of the label data of specimens from the Australian Commonwealth, state and territory herbaria. The success of AVH ultimately led to funding for the Atlas of Living Australia (ALA) and in October 2012 AVH was re-launched as part of the ALA infrastructure (http://avh.chah.org.au). Since 2012, some university herbaria have also joined AVH and in June 2017 the New Zealand Virtual Herbarium (NZVH) was integrated, giving rise to the Australasian Virtual Herbarium. The AVH currently holds almost 6.3 million records from 23 Australian and New Zealand herbaria and comprises over 80 per cent of the vouchered occurrence records of Australian plants, algae and fungi in ALA.

While no longer at the forefront of the digitisation wave, having been around for so long, $\mathrm{AVH}$ can provide valuable insights into how and to what extent herbarium data is used and into the benefits of digitisation for herbaria and natural history collections. Six years of download statistics shows an ever-increasing use of AVH data and a widening of the user base. A far greater proportion of downloaded records is ostensibly used for new uses like ecological research and conservation, than for the traditional uses of herbarium specimen data, systematics and collections management. The last few years have also seen an increase in the use of AVH data in education. Over the years many research articles based on AVH data have been written, mostly at the interface between ecology and systematics, i.e. biogeography and conservation science.
\end{abstract}


With the increase of the types of uses for herbarium data, it becomes important, also for the herbaria themselves, to consider the fitness for purpose of AVH data for uses other than the purpose for which it was collected. It has become apparent that the uncertainty of the latitudes and longitude provided with herbarium specimens is often too great for use in ecological research or conservation and there is a bias in collection density at the bioregion level, which is likely to be more severe at smaller spatial scales. Also, while the demand for vouchered occurrence records is increasing, we have seen a steady decrease in the intake of new specimens by Australian herbaria since the 1980s.

This presentation will cover how Australian herbaria use AVH, how other users use AVH and what being part of AVH has meant to Australian herbaria.

\section{Keywords}

herbarium data, digitisation, usage

\section{Presenting author}

Niels Klazenga 